\title{
La competencia intercultural en las organizaciones: una aproximación teórica
}

\author{
Carmen Elboj Saso \\ celboj@unizar.es \\ Universidad de Zaragoza \\ Diana Valero Errazu \\ dvalero@unizar.es \\ Universidad de Zaragoza \\ Tatiana Iñiguez Berrozpe \\ tatanai@unizar.es \\ Universidad de Zaragoza \\ Carlos Gómez Babillo \\ cgomez@unizar.es \\ Universidad de Zaragoza
}

Resumen: Cada vez son más las organizaciones que reflejan la multiculturalidad en alguna de sus facetas. La necesidad de que las relaciones que se establecen entre ellos sean exitosas es un imperativo organizativo. Pese a ello, las investigaciones demuestran un amplio fracaso en estos ámbitos, por lo que las empresas están acudiendo a consultorias para obtener formación que faculte a sus trabajadores para desempeñarse con éxito en estas relaciones multiculturales, es lo que se ha denominado competencia intercultural. El presente artículo aborda este concepto aplicado a las organizaciones, concluyendo la necesidad de ampliar la formación universitaria en este aspecto.

Palabras clave: Competencia intercultural; competencias; multiculturalismo; educación; educación superior. 
INTERCULTURAL COMPETENCE IN ORGANIZATIONS: A THEORETICAL APPROACH

Abstract: Nowadays, there are more and more organizations that reflect multiculturalism in some of their characteristics. The need for the relationships established between them to be successful is an organizational imperative. Despite this, research shows a wide failure in these areas, so that companies are going to consultants to obtain training that empowers their workers to play successfully in multicultural relations, is what has been called intercultural competence. This article addresses this concept applied to organizations, concluding the need to expand university education in this regard.

Key words: Intercultural competence; competences; multiculturalism; education; bigher education. 


\section{Introducción: Globalización y mercado de trabajo}

El factor multicultural en los mercados no ha dejado de crecer en los últimos años, especialmente de la mano de la globalización y, recientemente, debido al aumento de las empresas ligadas al desarrollo de Internet. Esto ha cambiado el panorama de las empresas. Por un lado, las empresas tratan de atraer talento desde cualquier parte del mundo, creando grupos de trabajo diversos en cuanto a su origen, religión, orientación sexual... Por otro lado, cada vez más empresas abren sedes en otros países o deslocalizan su producción. Ambos procesos implican que culturas diferentes, bien organizacionales, bien propias de cada país, deben trabajar conjuntamente para lograr unos determinados objetivos, lo que en muchos casos no resulta sencillo. Esta capacidad para moverse adecuadamente entre las diferentes culturas es lo que se conoce como competencia intercultural, y cada vez está recibiendo más interés por parte de la investigación internacional (Johnson, Lenartowicz, \& Apud, 2006; Keršienė \& Savanevičiené, 2015).

En el presente artículo abordamos esta competencia, su origen, su aplicación específica a las organizaciones y cómo se puede incrementar dicha competencia para aumentar el éxito de las cada vez más numerosas organizaciones multiculturales.

\section{La competencia intercultural: terminología y caracte- rización}

Dos conceptos que resultan confusos son los de multicultural e intercultural, por lo que resulta importante aclarar su relación desde el principio. Cuando hablamos de multicultural hacemos referencia a un contexto culturalmente diverso, es decir, un espacio en el que se encuentran miembros de diferentes culturas. En este sentido, espacio se entiende en sentido amplio, pudiendo hacer referencia a un país, una sociedad, una empresa o un mercado de trabajo determinado. Multicultural es un término que describe una determinada situación, refleja una pluralidad existente independientemente de las relaciones que se den entre los individuos que se encuentran en dicho espacio. Este término surge en la década de los 60 en los países anglosajones, en un marco de lucha por los derechos civiles, por los derechos de las minorías (todavía existían escuelas segregadas) y de las mujeres, la guerra de Vietnam, etc.

Sin embargo, cuando se utiliza el término intercultural hacemos referencia a cómo son las relaciones, las interacciones que se dan y que se establecen entre las personas de las diferentes culturas. La interculturalidad implica una voluntad, 
una dirección hacia la cual queremos orientar las relaciones entre las culturas siendo esta dirección la coexistencia y la convivencia (Beltrán, 2002).

La interculturalidad supone mirar la multiplicidad de formas de vida de los grupos sociales y reconocer que la propia cultura y la del otro cumplen una función social, como es "expresar emociones, deseos, modos de sentir el mundo... enseña valores, permite preferencias y elecciones de fines, da sentido a actitudes y comportamiento; al hacerla presta unidad a un grupo, integra a las personas en un todo colectivo... determina criterios para la elección de los medios adecuados para la realizar estos fines y valores" (Villoro, 2007: 139).

Este fenómeno de interrelación entre culturas diferentes ha dado lugar a una serie de términos también ambiguos y vagos, sujetos a diferentes interpretaciones, como aculturación, transculturación, interculturalidad, entre otros. Se trata de explicar la manera en que por el contacto cotidiano entre grupos, de orígenes históricos distintos, ocurren las transformaciones sociales y se producen cambios en las mentalidades, en los universos simbólicos, en el imaginario de las personas, en su forma de sentir y percibir el mundo y, en especial, en su manera de acercarse y enfrentarse a situaciones nuevas, de relacionarse con datos culturales distintos a los propios. La diversidad cultural se presenta en espacios definidos donde coexisten grupos humanos con tradiciones culturales diferentes.

La interculturalidad implica la actitud de asumir positivamente la situación de diversidad cultural en la que uno se encuentra. A nivel individual, nos referimos a la actitud frente a las influencias culturales a las que estamos expuestos, a veces contradictorias entre sí o por lo menos no siempre fáciles de armonizar. La interculturalidad como principio rector orienta también procesos sociales que intentan construir, sobre la base del reconocimiento del derecho a la diversidad y en contra de todas las formas de discriminación y desigualdad social, relaciones dialógicas y equitativas entre los miembros de universos culturales diferentes.

Este concepto de interculturalidad no ha calado en la cultura anglosajona que, para hacer referencia a relaciones entre culturas utiliza el concepto de cross-cultural, definido como la habilidad de los individuos para funcionar efectivamente con miembros de otra cultura (Gertsen, 1990).

La amalgama de nomenclaturas que se utiliza para referirnos a la competencia intercultural — en España - o competencia cultural —en países anglosajones contribuye a la confusión existente respecto a este desempeño, por lo que antes de centrarnos específicamente en el ámbito de las organizaciones vamos a abordar brevemente el origen de este concepto.

El constructo de la competencia comunicativa intercultural se aborda inicialmente a finales de la década de los 80 , principios de los 90 , por varias discipli- 
nas, entre ellas la psicología, la educación y la gestión, pero partiendo del trabajo previo en el ámbito de la comunicación. El punto de partida fue que el enfoque comunicativo puso en evidencia que para comunicarse en un idioma no basta con tener los conocimientos de una determinada lengua, también es preciso tener la habilidad de usarla. Paralelamente se dio un intento de establecer esta misma diferenciación en el ámbito de la cultura. Se trata no solo de adquirir conocimientos sobre una determinada cultura, sino de saber utilizarlos para desenvolvernos adecuadamente en ella. En este sentido, en el contexto americano se parte de la competencia comunicativa, cuyas dos características esenciales son la eficacia y la adecuación. "La eficacia es el logro exitoso de metas, objetivos o recompensas valoradas en relación con los costos. La adecuación significa que las reglas, normas y expectativas valiosas de la relación no se violan significativamente" (Spitzber, 1991:354). Es decir, no basta con saber gramaticalmente un idioma - eficacia-, sino que es preciso saber utilizarlo adecuadamente y siguiendo los usos y costumbres del contexto en el que se da la comunicación - adecuación- (Cheng y Satarosta, 1996). Por tanto, la competencia comunicativa intercultural, además de al idioma, está estrechamente ligada a la cultura pero, ¿qué es cultura?, ¿Cómo enseñamos esa cultura? Inicialmente se partía de la idea de que era necesario conocer las características de la cultura-meta, hechos significativos etc. contraponiendo la cultura nativa con la extranjera. Actualmente esta visión de la cultura como información, ha sido tildada de reduccionista y simplista y se ha evolucionado hacia una visión que aboga por la de entender la cultura como algo no estático, cambiante, donde las personas pueden tener identidades múltiples y redes transnacionales y que, por tanto, no se pueden reducir a una enumeración de características de una determinada cultura (Kramsch, 2001).

Por otra parte, la competencia comunicativa se da en relación a los otros y es por tanto interpersonal; la interculturalidad viene dada por el contexto, en sentido amplio, en el que se da dicha comunicación (Cheng y Satarosta, 1996). En este sentido, la competencia comunicativa intercultural debe tener en cuenta que el comportamiento sea apropiado y efectivo en un determinado contexto, entre unas determinadas personas (Lustig y Koester, 1996; Spitzberg, 2000). Así, tenemos dos partes al referirnos al factor cultural. Por un lado, "los aspectos socioculturales de la lengua (que incluyen las variedades lingüísticas, el comportamiento comunicativo y los significados socioculturales del vocabulario); y por otro, la realidad sociocultural de los hablantes como contexto en el que la lengua se usa y cobra sentido" (Areizaga, 2001:162). Respecto a esta segunda parte hablaremos de competencia comunicativa intercultural para referirnos a aquella interacción que se da entre personas con un background diferente y que, por tanto, 
pueden tener un esquema de valores, comportamientos o actitudes diversos. En este sentido es importante tener en cuenta que la visión del mundo de cada individuo es cambiante y viene determinada por el espacio en el que se ha socializado, no solo por una cuestión de espacio físico y temporal, sino también, y fundamentalmente, por las interacciones que ha tenido en el camino, las cuales le han hecho construirse un esquema de valores mediante el cual da respuesta a las cuestiones de su vida cotidiana. Volveremos a esta cuestión más adelante. En el caso de las personas que se trasladan a otros países, si su esquema previo no coincide con el esquema mayoritario en el nuevo país es cuando se habla de "choque de culturas"," disonancia cultural", "éxito/fracaso cross-cultural" etc. (Koester et alii, 1993). Éste es uno de los factores que, como se desarrolla más adelante, se consideran necesarios a la hora de desarrollar la competencia intercultural, ser conscientes de nuestras propias vías, prejuicios y esquemas de valores (Zabalza, 1992).

Así, Meyer (1991) define la competencia comunicativa intercultural «como parte de una amplia competencia del hablante de una lengua extranjera, identifica la habilidad de una persona de actuar de forma adecuada y flexible al enfrentarse con acciones, actitudes y expectativas de personas de otras culturas. La adecuación y la flexibilidad implican poseer un conocimiento de las diferencias culturales entre la lengua extranjera y la propia; además, tener la habilidad de solucionar problemas interculturales como consecuencia de dichas diferencias" (Meyer, 1991:137)

A pesar de los esfuerzos llevados a cabo por las diferentes disciplinas científicas, todavía no se ha alcanzado una definición general de competencia intercultural y por tanto, tampoco existe un modelo empírico validado, aunque sí que hay un grado de acuerdo amplio en cuanto a las dimensiones que forman parte de la competencia intercultural (Fritz, Möllenberg, \& Chen, 2002; Müller \& Gelbrich, 2001; Chen \& Starosta, 1997; Chen \& Starosta, 1999). En cuanto a las dimensiones de la propia competencia intercultural, la propuesta más aceptada es la que parte del trabajo de Sue et alii, (1982) que divide las competencias en 3 categorías: (A) el componente de actitudes / creencias - una comprensión del propio condicionamiento cultural que afecta a las creencias, valores y actitudes personales; (B) componente cognitivo - comprensión y conocimiento de las cosmovisiones de individuos y grupos culturalmente diferentes; $y(C)$ componente de habilidades uso de habilidades de intervención / comunicación culturalmente apropiadas. Esta división de tres dominios se actualizó más tarde en una matriz de $3 \times 3$ lo que da como resultado 31 competencias (Sue et alii, 1992). Partiendo de este trabajo, Chen \& Starosta, (1996) proponen 3 dimensiones de la competencia intercultural: la dimensión afectiva (sensibilidad intercultural), la cognitiva (conciencia 
intercultural) y la comportamental (habilidades interculturales) y que han sido ampliamente aceptadas (Bennett, 2001; Bradford, Allen, y Beisser, 1998; Graf, 2004; Howard-Hamilton, 2003; Spitzberg, 2000; Ting-Toomey, 1999, entre otros). Aunque algunos estudios defienden que este modelo no tiene en cuenta otros factores importantes que deberían ser incluidos, como sería la identidad étnica (Ponterotto, Rieger, Barrett, \& Sparks, 1994; Ponterotto, Gretchen, Utsey, Rieger y Austin, 2002; Vinson \& Neimeyer, 2000).

Tabla 1: Elementos de la competencia intercultural

\begin{tabular}{|c|c|c|}
\hline $\begin{array}{c}\text { Dimesiones } \\
\text { (Chen \& Starosta, 1996; Sue, } \\
\text { 1982) }\end{array}$ & Qué es & $\begin{array}{l}\text { Elementos de cada dimensión } \\
\text { (Chen \& Starosta, 1996) }\end{array}$ \\
\hline \multirow{4}{*}{$\begin{array}{l}\text { intercultural sensitivity - componen- } \\
\text { te emocional o afectiva }\end{array}$} & \multirow{4}{*}{$\begin{array}{l}\text { Incluye el conjunto de capacida- } \\
\text { des que nos permiten controlar las } \\
\text { emociones negativas ante los pro- } \\
\text { cesos multiculturales y promover } \\
\text { las reacciones positivas hacia los } \\
\text { mismos }\end{array}$} & Auto-concepto \\
\hline & & Mente abierta \\
\hline & & Actitud no prejuiciosa \\
\hline & & $\begin{array}{l}\text { Relajación social, sentirse cómodo } \\
\text { en las relaciones sociales intercul- } \\
\text { turales }\end{array}$ \\
\hline \multirow{2}{*}{$\begin{array}{l}\text { intercultural awareness - } \\
\text { Componente cognitivo }\end{array}$} & \multirow{2}{*}{$\begin{array}{l}\text { Es la conciencia de las diferencias } \\
\text { culturales }\end{array}$} & Conciencia propia \\
\hline & & Conciencia cultural \\
\hline $\begin{array}{l}\text { Intercultural adroitness - } \\
\text { Componente comportamental, ha- } \\
\text { bilidades }\end{array}$ & $\begin{array}{l}\text { Es la capacidad de un individuo de } \\
\text { hacer el trabajo y lograr los objeti- } \\
\text { vos comunicativos en las interaccio- } \\
\text { nes multiculturales }\end{array}$ & $\begin{array}{l}\text { Gestión de las identidades, de las } \\
\text { interacciones, habilidad comunica- } \\
\text { tiva, comportamiento flexible, culti- } \\
\text { vo de las relaciones... (Chen, 2002). }\end{array}$ \\
\hline
\end{tabular}

Fuente: Chen \& Starosta, 1996; Sue, 1982. Elaboración propia.

\section{La competencia cultural en las organizaciones}

Hasta ahora hemos hecho referencia a la competencia comunicativa intercultural desde el ámbito de la comunicación, pero no ha sido el único que lo ha abordado, de hecho, ha sido criticado desde otros ámbitos. Por ejemplo, desde la antropología se ha hecho referencia a que la propia forma de entender la cultura por parte de la investigación en comunicación intercultural ha hecho que construyan esas diferencias que están tratando de superar Koch, G. (2009:1).

Precisamente porque la competencia intercultural se desarrolla en el marco de la competencia comunicativa, muchas de las investigaciones que se han llevado a cabo (Vilaseca, 2000; Trujillo Saez, 2002; Paricio, 2004) se han ligado al aprendizaje y uso de la L2 (segunda lengua o lengua extranjera) por parte del alumnado, siendo el ámbito educativo uno de los ámbitos en los que las investigaciones sobre competencia intercultural se han desarrollado más.

Otro de los ámbitos en los que se ha estudiado ha sido desde el mercado, es decir, desde la gestión. Como hacíamos referencia en nuestra introducción, 
el mercado, tanto productivo como laboral, ha cambiado con la globalización y muchos de los negocios han experimentado dicha globalización mediante la interacción con clientes, competidores, suministradores o trabajadores internacionales (Alon y Higgins, 2005). Cada vez son más las empresas que tienen relación con mercados internacionales, bien porque tienen su producción descentralizada, porque compran sus productos fuera, porque tienen sedes en otros países, venden sus productos internacionalmente, su plantilla de trabajadores es de distinta procedencia geográfica y/o cultural, etc. Son infinidad las situaciones que hacen evidente la necesidad de tener una serie de competencias personales que respondan a estos retos. Entre ellas, se incluyen la flexibilidad, adaptabilidad, tolerancia, conciencia de la propia cultura, etc. (Cant, 2004). En resumen, lo que se ha llamado una elevada competencia intercultural.

Específicamente en este ámbito, al igual que sucede en los demás, no existe una definición unificada y aceptada de este tipo de aptitud. Aeneas (2009) la define como "un conjunto holístico y aplicado de componentes cognitivos, actitudinales y comportamentales que permiten apreciar y dar respuesta a los requerimientos del trabajo y de relación generados por la diversidad cultural de la organización" (Aneas, 2009:11). Desde este ámbito, también se ha entendido como parte de la inteligencia cultural de los individuos que tienen que trabajar en estas situaciones multiculturales (Earley \& Mosakowski, 2004; Earley, 2002). Esta habilidad tiene al menos cuatro componentes (Ang, Dyne, Koh, Ng, Templer, Tay, \& Chandrasekar, 2007: 6-7):

- Inteligencia metacognitiva. La capacidad de adquirir conocimientos culturales, reconocer las suposiciones culturales, comprender las normas culturales y percibir las preferencias culturales de los demás antes y durante las interacciones.

- Inteligencia cognitiva: Conocimiento de los sistemas económicos, legales, y sociales, en diferentes culturas y subculturas.

+ Inteligencia motivacional: El deseo de aprender y funcionar en situaciones que involucren diferencias culturales, basadas en el interés intrínseco y la confianza en la capacidad de uno para tratar con ellos.

- Inteligencia del comportamiento: La capacidad de exhibir acciones verbales y no verbales apropiadas a la situación, incluyendo palabras, tono, gestos y expresiones faciales, al interactuar con personas de diferentes culturas.

Sin embargo, otros autores han ido más allá y han dicho que lo fundamental es saber orientar dichos conocimientos, habilidades y atributos hacia el logro de objetivos, proponiendo una definición específica que señala que "la competen- 
cia intercultural en los negocios internacionales es la efectividad de un individuo en aprovechar un conjunto de conocimientos, habilidades y atributos personales para trabajar con éxito con personas de diferentes orígenes culturales nacionales en el país o en el extranjero" (Johnson, Lenartowicz y Apud (2006:530).

Esta apreciación coincide con la categorización más reciente del de Modelo competencias interculturales, y que se compone de dos macro competencias: el diagnóstico (D), que coincidiría con la mayoría de las definiciones de competencia intercultural, y que fundamentalmente consiste en ser consciente de cómo afecta la multiculturalidad a los procesos y a la propia organización; y el afrontar (A), que estaría más en la línea de la actuación, de combinar los conocimientos, habilidades y actitudes hacia la resolución de problemas (Aneas, 2009).

Este modelo propuesto por Aeneas (2003) señala que "la persona competente interculturalmente, hace un diagnóstico de su escenario profesional multicultural, atendiendo a los aspectos sociales, funcionales y contextuales y es capaz de relacionarse, comunicándose, negociando y trabajando con personas de cultura diferente a la propia y es capaz de hacer frente a los varios retos y situaciones que se deriven de la naturaleza multicultural del ámbito laboral. Obviamente, al diagnosticar, relacionarse y afrontar situaciones problemáticas, la persona competente interculturalmente integra y aplica las dimensiones cognitivas, afectivas y comportamentales, propias de la competencia intercultural" (Donoso, Rodríguez, Aneas, De Santos y Curós, 2009:153).

Otra de las cuestiones es en qué ámbitos dentro de una organización pueden darse interacciones interculturales. En este sentido Ayoko, Hartel, Fisher y Fujimoto (2004) hacen referencia a tres niveles, trabajadores de la misma organización, clientes dentro de la cultura local y, finalmente, con clientes fuera de la cultura local. La primera serían interacciones que se dan dentro de la organización con trabajadores que pueden ser de la misma o de diferente cultura; el segundo nivel incluiría a los proveedores de servicios en la misma cultura/país y la prestación de servicios fuera de la cultura/país. Es en este último nivel en el que se han detectado mayores problemas. En la revisión llevada a cabo por Johnson, Lenartowicz y Apud (2006) se hace referencia a que "dos temas generales emergen de la literatura: el fallo en las expatriaciones, y una amplía inhabilidad de los directivos para apreciar los retos culturales de hacer negocios más allá de tus fronteras" (Johnson, Lenartowicz \& Apud, 2006: 525).

En este sentido, los mismos autores hacen referencia a que la conceptualización de la competencia intercultural, hasta ahora, no ha tenido en cuenta un factor tan importante como es el contexto en el que se da, ya que la mayor parte de las investigaciones, - especialmente las del ámbito de la psicología o la educa- 
ción-, están referidas a trabajadores, clientes o estudiantes que se encuentran en el país, es decir, que no se trasladan. Sin embargo, en las negociaciones internacionales, los fallos se dan cuando los trabajadores van a otras culturas y no tanto entre trabajadores de diferentes culturas dentro de una empresa. Concretamente señalan que "los individuos que poseen las habilidades, los conocimientos y los atributos necesarios que les permitan desempeñarse eficazmente en un entorno intercultural pueden todavía tener dificultades para lograr un alto nivel de eficacia en competencia intercultural frente a las barreras contextuales que desafían las operaciones comerciales internacionales. Estas incluyen, pero no se limitan a, los condicionantes físicos, económicos, políticos y legales con los que un gerente expatriado puede encontrarse" (Johnson, Lenartowicz \& Apud, 2006: 532). Entre los condicionantes o limitaciones contextuales, se incluyen el etnocentrismo institucional (Hofstede, 2001) -la institución trata de mantener sus usos y costumbres independientemente de la cultura en la que se encuentre-, la distancia cultural (Shenkar, 2001) - que aborda la diferencia entre el país de origen y sus afiliados/trabajadores en el extranjero- y finalmente la policontextualidad (Von Glinow, Shapiro y Brett, 2004) - espacio en el que se dan diferentes convenciones contextuales al mismo tiempo-.

Si bien en la mayoría de estos casos estaríamos refiriéndonos a individuos dentro de una organización, y no propiamente a las organizaciones, concretamente en el ámbito de estas, se hace referencia a la necesidad de abogar por un desarrollo organizacional multicultural y también se divide en tres estadios (Sue, 2001: 807).

1. Organizaciones monoculturales. En este extremo están organizaciones fundamentalmente eurocéntricas y etnocéntricas. Operan desde los siguientes supuestos: A) Hay una exclusión implícita o explícita de las minorías raciales, mujeres y otros grupos marginados; (B) están estructuradas para la ventaja de la mayoría euroamericana; $(\mathrm{C})$ entienden que sólo hay una manera de prestar atención médica, enseñar o administrar; (D) se cree que la cultura tiene un impacto mínimo en la administración, la salud o la educación; (E) los clientes, trabajadores o estudiantes deben asimilarse a la cultura mayoritaria (F) otras formas culturales de hacer no son ni reconocidas ni valoradas; $(\mathrm{G})$ todos deben ser tratados de la misma manera, independientemente de sus características; y $(\mathrm{H})$ existe una fuerte creencia en el concepto del crisol.

2. Organizaciones no discriminatorias. A medida que las organizaciones entienden la relevancia de la cultura y se vuelven más receptivas a ella, entran en una etapa no discriminatoria. En esta etapa se caracterizan por: (A) Poseen políticas y prácticas inconsistentes con respecto a temas multiculturales. Ciertos departa- 
mentos y algunos trabajadores son sensibles a las cuestiones de las minorías, pero no es una prioridad organizativa. (B) El liderazgo puede reconocer la necesidad de alguna acción, pero carecen de un programa o política que aborde la cuestión de los prejuicios. (C) Se da un intento de hacer que el clima o los servicios de una organización sean menos hostiles, pero estos cambios son superficiales y a menudo sin convicción. Es más probable que estén presentes por razones de relaciones públicas que por una verdadera vocación de cambio. (D) La igualdad de oportunidades para el empleo, la acción afirmativa y la simetría numérica de las minorías y las mujeres se aplican a regañadientes.

3. Organizaciones multiculturales. A medida que las organizaciones se vuelven más multiculturales, empiezan a valorar la diversidad y continúan implementando y afianzando los cambios culturales. Su manera de operar refleja estos valores: (A) Están en el proceso de trabajar en una visión que refleje el multiculturalismo; (B) Las contribuciones de los diversos grupos sociales y culturales se reflejan en la misión, operaciones, productos y servicios de la empresa; (C) se valora el multiculturalismo y es visto como un activo; (D) Abrazan activamente la visión, planificación y actividades de resolución de conflictos que permitan igualdad de acceso y oportunidades; (E) Son conscientes de que la igualdad de acceso y las oportunidades no son igualdad de trato; y $(F)$ trabajan para diversificar el entorno.

\section{Formación para la competencia intercultural}

La cuestión más compleja de la competencia intercultural es cómo abordamos su aprendizaje. Cuando hablamos de diferencias interculturales la diferencia más evidente es el idioma, pero como ya hemos señalado el cómo utilizar adecuadamente dicho idioma va de la mano de la cultura, tanto de los interlocutores como de la organización y/o país al que nos refiramos. La cuestión es, en este sentido, cómo aprender esa cultura y cuáles son los elementos o componentes que influyen en ello.

En la primera sección del presente artículo hacíamos referencia a que la competencia intercultural está formada por conciencia, conocimientos y habilidades (Howard-Hamilton, 2003). Son, estos dos últimos, los elementos sobre los que se debe trabajar mediante la formación, para lograr modificar el nivel de competencia intercultural del que parte un determinado individuo. La conciencia se modularía a través de la experiencia individual de cada persona.

Sin embargo, en el ámbito de las empresas internacionales, se ha hecho referencia a que no basta con estas tres competencias ya que eso no implica, necesariamente, que se obtengan los resultados deseados; es preciso estar motivado y tener la capacidad de adaptar todo ello a los nuevos contextos (Earley, 2002). 
En este sentido, todos los autores parten de la idea de que la competencia intercultural se adquiere $y$, por tanto, puede ser enseñada y promovida, aumentándola con el tiempo, pero también depende de factores personales hasta qué nivel se puede llegar (Hofstede, 2001).

Concretamente, Meyer (1991) propone tres etapas en la adquisición de la competencia intercultural:

- Nivel monocultural: en este nivel la persona se basa referencialmente en su cultura. La cultura extranjera se ve y se interpreta según la perspectiva de la propia cultura. Prevalecen los tópicos, prejuicios y estereotipos

- Nivel intercultural: la persona está mentalmente situada entre las dos culturas. El conocimiento que tiene de la cultura extranjera le permite hacer comparaciones entre ambas y posee suficientes recursos para explicar las diferencias culturales.

- Nivel transcultural: la persona se sitúa por encima de las culturas implicadas, con una cierta distancia, permitiéndole colocarse en una situación de mediador entre ambas. Cada uno se aleja de los estilos habituales de mirar su entorno para poder así adoptar puntos de vista ajenos, sin renunciar a la propia identidad cultural.

Para pasar de una etapa a otra se han desarrollado diferentes modelos de aprendizaje en función de a quién van dirigidos (estudiantes o profesionales) y con qué objetivos. Nos referiremos aquí a los dos modelos más aplicados en el ámbito de la gestión, la formación intercultural (Brislin \& Yoshida,1994) y la formación para la diversidad (Cox, 2001). Aeneas (2010:436).

En cuanto a la primera, la formación intercultural, está indicada para aquellos trabajadores que tienen que desarrollar su labor en otras culturas. El hecho de que una determinada organización envíe a un empleado a trabajar fuera de sus fronteras no implica que dicho empleado esté preparado para ello, por lo que es necesaria una formación previa que tiene un carácter puntual y es específica y de corta duración. Es una formación que se da a los trabajadores o directivos que se van a un determinado país, por lo que está impartida por profesorado con un profundo conocimiento de la cultura-meta, generalmente de institutos privados o consultorías, e incluyen conocimientos generales, específicos, historia, política, economía, etc. del país al que van a trasladarse los trabajadores así como aspectos de la cultura diaria (Juárez Medina, 2005)

Las críticas a este enfoque se han centrado en 4 ámbitos: (1) asumen que todos los individuos necesitan el mismo tipo de entrenamiento; (2) los métodos están centrados fundamentalmente en el componente cognitivo, por lo que no enseña actividades metacognitivas necesarias para lograr los objetivos; (3) se pre- 
supone un vínculo entre los valores culturales, las normas y los individuos, lo que no siempre responde a la realidad; (4) el aprendizaje es muy analítico, asumiendo que el alumno es capaz de aplicar los conocimientos a las nuevas situaciones a las que se enfrenta, lo que no está demostrado si no se enseñan las estrategias metacognitivas necesarias (Earley y Peterson, 2004). Por otra parte, específicamente sobre los logros que obtienen este tipo de formaciones, Sercu (2002) señala que para lograr verdaderos cambios es precisa una formación más continuada.

Para superar estas limitaciones, y siguiendo el enfoque de la inteligencia cultural anteriormente citado, Earley y Peterson (2004) proponen una formación intercultural que "consta de tres elementos fundamentales: meta-cognición y cognición (pensamiento, aprendizaje y elaboración de estrategias); motivación (eficacia y confianza, persistencia, congruencia de valor y afecto para la nueva cultura); y comportamiento (mimetismo social, y repertorio conductual)" (ídem:105).

Por otra parte, la formación para la diversidad tiene también importantes puntos débiles, como es la escasa evaluación de la efectividad de estos programas (Jackson, Joshi, \& Erhardt, 2003). Frente a la propuesta de la educación intercultural, la formación para la diversidad comienza con una evaluación individual, lo que permite ajustar el curso a los participantes está más enfocada en la adquisición y desarrollo de competencias orientadas hacia el logro de objetivos y tienen en cuenta el contexto en el que están situados (Earley y Peterson, 2004). Aunque, nuevamente, se centra en el aprendizaje explícito, por lo que Avery y Thomas (2004) señalan la necesidad de que los cursos sobre diversidad incluyan no solo el conocimiento explícito, sino también el implícito mediante estrategias como la autoevaluación, el aprendizaje experimental y el contacto entre grupos diversos.

En cualquier caso, específicamente para la enseñanza de la competencia intercultural en el marco de los negocios internacionales, como hemos señalado, la mayoría de la formación se lleva a cabo a través de consultorías especializadas, lo que supone per se una limitación. En este sentido Egan y Bendick (2008) hablan de la necesidad de que los profesores vayan más allá de las fronteras marcadas por su disciplina a la hora de entender la cultura de una manera estática y homogénea, o que presenta a los otros como algo exótico. Este trabajo entre disciplinas, aunque complejo, es fundamental para desarrollar adecuadamente una caracterización de la competencia intercultural que sea aplicable a otras disciplinas y que responda a un concepto tan multifacético. 


\section{Conclusiones}

Cada vez más voces señalan la importancia de la competencia intercultural en el ámbito empresarial, haciendo referencia al aumento cada vez mayor del flujo de trabajadores transfronterizos, empresas multinacionales y multiculturales en las que empleados con un background diferente deben trabajar en equipo para lograr unos resultados, o en las que un trabajador es trasladado a otro país para poner en marcha una nueva sede, por ejemplo (Johnson, Lenartowicz, \& Apud, 2006; Keršienè \& Savanevičienè, 2015). Esto no implica necesariamente que los miembros de la organización, o la organización en sí misma, esté preparada para abordar los retos que dicha multiculturalidad implica.

En este sentido, es cada vez más demandada en las organizaciones una elevada competencia intercultural pero, como hemos indicado, este concepto todavía no está suficientemente desarrollado por lo que la formación en este ámbito todavía requiere de un profundo impulso y perfeccionamiento.

\section{Bibliografía}

Ang, S.; Dyne, L. V;; Koh, C.; Ng, K.; Templer, K. J.; Tay, C., \& Chandrasekar, N. A. 2007. Cultural intelligence: Its measurement and effects on cultural judgment and decision making, cultural adaptation, and task performance. Singapore: Nanyang Technological University.

Alon, I., \& Higgins, J. M. (2005). “Global leadership success through emotional and cultural intelligences". Business horizons, 48(6), 501-512.

Aneas, A. (2003). “Competencias interculturales transversales: un modelo para la detección de necesidades de formación". Universidad de Barcelona (tesis doctoral). (URL): <http://www.tdcat.cesca.es/TESIS_UB/AVAILABLE/ TDX-1223104-122502//0.PREVIO.pdf $>$.

- (2004). Competencia intercultural, concepto, efectos e implicaciones en el ejercicio de la ciudadanía. Barcelona: Universidad de Barcelona.

- (2009). “Competencias interculturales transversales: su diagnóstico en equipos de trabajo de baja cualificación". Revista de investigación educativa, 27(1), 105-123.

Aneas, M. A. (2010).“Formación intercultural en las organizaciones. Bases para diseños formativos orientados al tratamiento de la interculturalidad en las organizaciones". Barcelona: Universidad de Barcelona. Disponible en: <http:// www.academia.edu/975698/Formaci\%C3\%B3n_intercultural_en_las_organizaciones_Bases_para_dise\%C3\%B1os_formativos_orientados_al_tratamiento_de_la_interculturalidad_en_las_organizaciones $>$. 
Areizaga, E. (2001). "Cultura para la formación de la competencia comunicativa intercultural: el enfoque formativo". Revista de Psicodidáctica, 11(12), $157-170$.

Avery, D., \& Thomas, K. (2004). "Blending Content and Contact: The Roles of Diversity Curriculum and Campus Heterogeneity in Fosterin Diversity Management Competency". Academy of Management Learning E Education, 3 (4), 380-396.

Ayoko, O;; Hartel, C.; Fisher, G. y Fujimoto, Y. (2004). “Communication competence in cross-cultural business interactions". En Tourish, Dennis and Hargie, Owen (ed), Key issues in organizational communication (pp.157171). Routledge, New York, NY.

BennetT, M. J. (2001). "Developing intercultural competence for global leadership". En R. D., and C. Fussinger (Eds.), Interkulturelles management: Konzeption - Beratung - Training (pp. 205-226). Wiesbaden: Gabler.

Bradford, L.; Allen, M., \& Beisser, K. (1998). "An Evaluation and MetaAnalysis of Intercultural Communication Competence Research". Obtenido de <http://files.eric.ed.gov/fulltext/ED417453.pdf $>$.

Brislin, R. W., \& Yoshida, T. (1994). Intercultural communication training: An introduction (Vol. 2). Sage Publications: Thousand Oaks, CA.

Cant, A. G. 2004. "Internationalizing the business curriculum: Developing intercultural competence". The Journal of American Academy of Business, September: 177-182.

Chen, G. M., \& Starosta, W. J. (1996). "Intercultural communication competence: A synthesis". En B. Burleson (Ed.), Communication Yearbook, 19 (pp. 353-383). Thousand Oaksः Sage.

- (1997). "A review of the concept of intercultural sensitivity". Human Communication, 1, 1-16.

- (1999). "A review of the concept of intercultural awareness". Human Communication, 2, 27-54.

- (2000)."The development and validation of the intercultural communication sensitivity scale". Human Communication, 3, 1-15.

Cox, T. Jr. (2001). “The multicultural organization”. En Albrecht, M.H. (Ed). International HRM. Managing diversity in the workplace. (pp. 245-260). Blackwell Publishers Ltd.

Donoso, T; Rodríguez, F. A. C.; Aneas, A.; De Santos, J., \& Curós, P. (2009). "Análisis de necesidades en formación intercultural en la administración pública". Revista de Investigación Educativa, 27(1), 149-167. 
EARLey, P. C. (2002). "Redefining interactions across cultures and organizations: Moving forward with cultural intelligence". Research in organizational behavior, 24, 271-299.

Earley, P. C., \& Mosakowski, E. (2004). Cultural intelligence. Harvard Business Review, 82(10): 1-9.

Earley, P. C. \& Peterson, R. S. (2004). "The elusive cultural chameleon: Cultural Intelligence as a new approach to intercultural training for the global manager". Academy of Management Learning and Education, 3 (1), 100-115.

EgAN, M. L., \& Bendick, M. (2008). “Combining multicultural management and diversity into one course on cultural competence." Academy of Management Learning E Education, 7(3), 387-393.

Fritz, W.; Möllenberg, A., \& Chen, G.-M. (2002)."Measuring intercultural sensitivity in different cultural contexts". Intercultural Communication Studies, $\mathrm{XI}(2), 165-176$.

Gersten, M.C. (1990). "Intercultural competence and expatriates”. International Journal of Human Resource Management, 1 (3), 241-62.

Graf, A. (2004). "Screening and training intercultural competencies: Evaluating the impact of national culture on intercultural competencies". International Journal of Human Resource Management, 15(6), 1124-1148.

Hofstede, G. (2001) Culture's Consequences: Comparing Values, Behaviors, Institutions, and Organizations Across Nations, 2nd edn, Sage Publications: Thousand Oaks, CA.

Howard-Hamilton, M.F. (2003) "Creating a culturally responsive learning environment for students of color". Working paper for Scholarship of Teaching and Learning Symposium. Bloomington, USA, 2003, October.

Jackson, S. E.; Joshi, A., y Erhardt, N. L. (2003). "Recent research on team and organizational diversity: SWOT analysis and implications". Journal of Management, 29 (6), 801-830.

Johnson, J. P;; Lenartowicz, T., \& Apud, S. (2006). "Cross-cultural competence in international business: Toward a definition and a model”. Journal of International Business Studies, 37(4), 525-543.

Juárez Medina, A. (2005). "Competencias lingüísticas e interculturales: tendencias actuales en las empresas alemanas". IDEAS-Revista electrónica, 2, 8-19.

Keršienė, K., \& Savanevičienė, A. (2015). "Defining and understanding organization multicultural competence". Engineering economics, 42(2), 45-52.

Kim, C. K., \& Chen, G. M. (1995). "Enhancing intercultural awareness through international e-mail debate". Report submitted to FIPSE, US Department of Education. 
Kramsch, C. (2001). "El privilegio del hablante intercultural". En M. Brram y M. Fleming. Perspectivas interculturales en el aprendizaje de idiomas. Enfoques a través del teatro y la etnografía (pp. 27-37). Madrid: Cambridge University Press.

Косн, G. (2009). Intercultural communication and competence research through the lens of an anthropology of knowledge. Forum Qualitative Sozialforschung/ Forum: Qualitative Social Research (Vol. 10, No. 1).

Koester, J.; Wiseman, R. L., \& Sanders, J. A. (1993). "Multiple perspectives for intercultural communication competence”. In R. L. Wiseman \& J. Koester (Eds.), Intercultural communication competence, Newbury Park, CA: Sage Publications, 3-15.

Lustig, M.W y Koester, J. (1996). Intercultural competence: Interpersonal communication across cultures. New York: Harper Collins.

Meyer, M. (1991): "Developing transcultural competente: casa studies of advanced foreign language learners". En D. Butjes y M. Byram (Eds.), Developing Languages and Cultures. Avon: Multilingual Matters Ltd., 136158.

Paricio, M. S. (2004). "Dimensión intercultural en la enseñanza de las lenguas y formación del profesorado”. Revista iberoamericana de educación, 34(4), 1-12.

Ponterotto, J. G.; Rieger, B. P.; Barrett, A., \& Sparks, R.(1994). “Assessing multicultural counseling competence: A review of instrumentation”. Journal of Counseling and Development. 72(3), 316.

Ponterotto, J. G.; Gretchen, D.; Utsey, S. O;; Rieger, B. P., \& Austin, R. (2002)."A revision of the multicultural counseling awareness scale". Journal of Multicultural Counseling and Development, 30(3), 153.

Sercu, L. (2002). "Autonomous Learning and the Acquisition of Intercultural Communicative Competence: Some Implications for Course Development". Language culture and curriculum, 15(1), 61-74.

Shenkar, O. (2001). "Cultural distance revisited: Towards a more rigorous conceptualization and measurement of cultural differences". Journal of international business studies, 32(3), 519-535.

Spitzberg, B.H., (1991). "Intercultural Communication Competence”. En R. Porter y L. Schneider, S. y J.-L. Barsoux (1997), Managing across borders. London: Prentice Hall.

- (2000). "A model of intercultural communication competence". En L. A. Samovar y R. E. Porter (Eds*), Intercultural communication: a reader. Belmont: Wadsworth. 
Sue, D. W*; Bernier, J. E*; Durran, A.; Feinberg, L.; Pedersen, P*; Smith, E. J., \& Vasquez-Nuttall, E. (1982). "Position paper: Cross-cultural counseling competencies". The counseling psychologist, 10(2), 45-52.

Sue, D. W.; Arredondo, P., \& McDavis, R. J. (1992). "Multicultural counseling competencies and standards: A call to the profession". Journal of Counseling $\mathcal{E}$ Development, 70(4), 477-486.

Sue, D. W. (2001). "Multidimensional facets of cultural competence". The counseling psychologist, 29(6), 790-821.

Ting-Toomer, S. (1999). Communicating across cultures. New York: Guilford.

VILÀ, R. (2003). "La competencia comunicativa intercultural en Educación Secundaria Obligatoria: Escala de sensibilidad intercultural”. En E. Soriano (Ed.), Perspectivas teórico-prácticas en educación intercultural. Almería: Publicaciones de la Universidad de Almería.

- (2006). "La dimensión afectiva de la competencia comunicativa intercultural en la Educación Secundaria Obligatoria: Escala de Sensibilidad Intercultural". Revista de investigación educativa, 24(2), 353-372.

Vilaseca, A. O. (2000). Hacia la competencia intercultural en el aprendizaje de una lengua extranjera: estudio del choque cultural y los malentendidos. Madrid: Editorial Edinumen.

Villoro, L. (2007). Los retos de la sociedad por venir: ensayos sobre justicia, democracia y multiculturalismo. México: Fondo Cultura Económica.

Vinson, T. S. \& Neimeyer, G. J. (2000). "The relationship between racial identity development and multicultural counseling competency". Journal of Multicultural Counseling and Development, 28(3), 177.

Von Glinow, M.A.; Shapiro, D.L. and Bret', J.M. (2004). "Can we talk, and should we? managing emotional conflict in multicultural teams". Academy of Management Review, 29(4), 578-592.

Zabalza, M. A. (1992). "Implicaciones curriculares de la educación intercultural". Bordón, 44(1), 45-58. 